Available online on 15.03.2017 at http://iddtonline.info
(c) 2011-17, publisher and licensee JDDT, This is an Open Access article which permits unrestricted
noncommercial use, provided the original work is properly cited

Review Article

\title{
THE ROLE OF ANGIOTENSIN II TYPE 2 RECEPTORS (AT2RS) IN THE REGULATION OF CARDIO-RENAL AND NEUROPROTECTIVE ACTIVITIES: POTENTIAL THERAPEUTIC IMPLICATIONS
}

\author{
Yalew Molla ${ }^{*}$, Mekonnen Sisay ${ }^{2}$ \\ ${ }^{1}$ Department of Pharmacy, College of Health Sciences, Debre Markos University, Gojam, Ethiopia \\ ${ }^{2}$ School of Pharmacy, College of Health and Medical Sciences, Haramaya University, Harar, Ethiopia
}

\begin{abstract}
Most of the physiological effects of the rennin-angiotensin system (RAS) are mediated by angiotensin II (AgII) type one receptors (AT1R), producing cellular dedifferentiation and proliferation; vasoconstriction; renal tubule sodium (Na+) reabsorption etc. However, the pathophysiologic role of AgII type two receptor (AT2R) has not been clearly defined yet. This review was, therefore, aimed at summarizing a plenty of primary literatures related to the role of AT2R. AT2R is a special G protein coupled receptor that is not coupled with the usual second messengers. The expression level of AT2R is greater in the neonates and fetal ages than in adults though its expression is up-regulated following tissue injury in adults implicating its role in regulating cell differentiation, growth and inflammations. Most of the cellular actions mediated by AT2R are counter regulatory to that of AT1R. AgII produces the cellular effects by acting on AT2R via different signal transduction pathways. The common cellular effects mediated by AT2R are antiproliferative, anti-inflammatory, vasodilation, natriuresis, etc which may be essential for modulating the cardiovascular, renal and brain injuries caused by different etiologies. The principal molecular signal transductions mediated by AT2R involve stimulation of the bradykinin and/or nitric oxide-cGMP pathwy, inactivation of mitogen activated protein kinase pathway by stimulating tyrosine and serine/threonien phosphatses, production of the neuroprotective factors, like BDNF, etc. These signaling pathways may exert cardio-renal and neuroprotective functions. Therefore, the development of drugs that stimulate the AT2R may the potential target to promote the treatment of different disorders related to the cardiovascular, renal and brain dysfunctions.
\end{abstract}

Keywords: Angiotensin II, AT2R, cardiovascular system, Renal system, neuroprotection

Article Info

Received 26 Nov 2016; Review Completed 23 Dec 2016; Accepted 09 March 2017, Available online 15 March 2017

Cite this article as:

Molla Y, Sisay M, The role of angiotensin II type 2 receptors (AT2RS) in the regulation of cardio-renal and neuroprotective activities: potential therapeutic implications, Journal of Drug Delivery and Therapeutics. 2017; 7(2):110-118

DOI: http://dx.doi.org/10.22270/jddt.v7i2.1356

*Address for Correspondence

Yalew Molla (Bpharm, MSc in Pharmacology), Department of Pharmacy, College of Health Sciences, Debre Markos University, Debre Markos, P.O.BOX, 269, Gojjam, Ethiopia, Cell phone, +251913-95-68-42, Email, yalew_molla@yahoo.com

\section{INTRODUCTION}

The renin-angiotensin system (RAS) is a coordinated hormonal cascade involved in cardiovascular system with AgII as the main effector peptide regulating blood pressure. ${ }^{1}$ Angiotensin peptides are derived from the precursor protein angiotensinogen via several enzymatic conversion pathways as shown in the figure -1 .
The RAS has different components to produce the active ligands; AgII, AgIII, Ag(IV), Ag (1-7), and Ag (3-7). These ligands interact with several receptor including AT1, AT2, AT4, and Mas distributed within the central and peripheral nervous systems as well as local RAS in several organs with different affinity and activities. $^{2}$ 


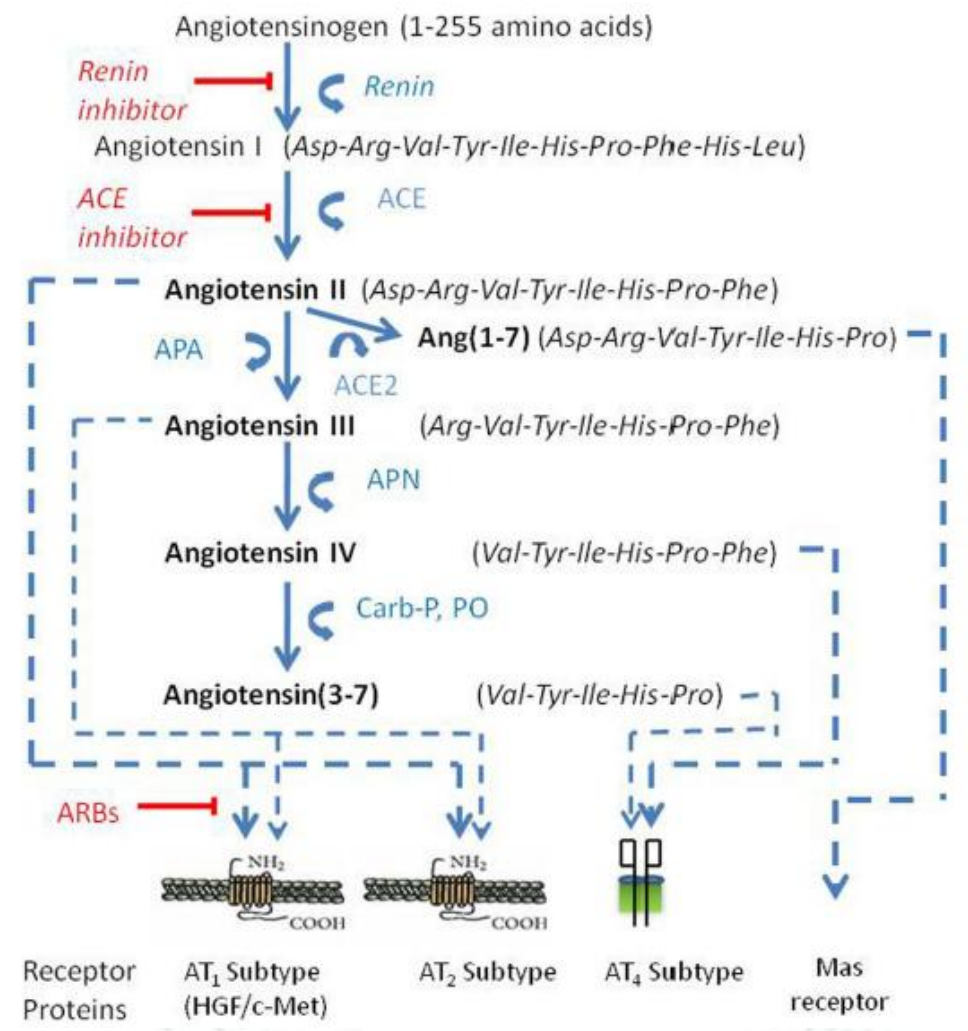

Figure 1: The renin-angiotensin pathway including active ligands (bold), enzymes, receptors, and inhibitors involved in the synthesis and ligand-receptor interaction in the different body systems. ${ }^{2}$ Abbreviations: ACE, angiotensin converting enzyme; ACE2, angiotensin converting enzyme 2; APA, aminopeptidase A; APN, aminopeptidase N; ARBs, angiotensin receptor blockers.

Ang II binds mainly two distinct RAS receptors; AT1R and AT2R, with high affinity. ${ }^{1,3}$ The vast majority of the physiological actions of Ang II are mediated by AT1Rs, including cellular dedifferentiation and proliferation; vasoconstriction; reduction of vascular compliance; cardiac contractility; increased renal tubule sodium $\left(\mathrm{Na}^{+}\right)$reabsorption; aldosterone, vasopressin and endothelin secretion; salt appetite; thirst; and activation of the sympathetic nervous system. ${ }^{1,3}$ But, the pathophysiologic role of AT2R is not yet clear; which is the main target of this review.

\section{AT2R Expression}

The AT2R is a 7-transmembrane GPCR composed of 363 amino acids with only $34 \%$ sequence homology with the $\mathrm{AT}_{1} \mathrm{R}$. $\mathrm{AT}_{2} \mathrm{Rs}$ are expressed ubiquitously at very high levels in the fetus, but decline dramatically in the neonatal period in most, but not all, tissues. Although there is relatively low expression of $\mathrm{AT}_{2} \mathrm{Rs}$ compared to $\mathrm{AT}_{1} \mathrm{Rs}$ in adult tissues, $\mathrm{AT}_{2} \mathrm{Rs}$ are expressed in the adult kidney, adrenal cortex, heart and vasculature, and predominate over AT1Rs in specific sites such as the uterus, ovary, and adrenal medulla and in discrete areas of the brain. ${ }^{1,3}$

Even though AT2R is much less abundant in adult tissues, it is up-regulated in pathological conditions such as myocardial infarction, stroke or cutaneous incisional wounds. ${ }^{3,4}$ This investigation was supported by an upregulation of $\mathrm{AT}_{2}-\mathrm{R}$ expression on fibroblast cells in failing hearts. ${ }^{5}$ AT2R present in the fibroblasts was shown to exert an inhibitory effect on Ang II-induced mitogen signals.
In addition, a study done on developmental changes in AT1R and AT2R-protein expression in rats using western blot technique showed that the expression of AT2R was lower in both fetuses and neonates than that in adults in brainstem, kidney and liver and the opposite expression level was true for AT1R. ${ }^{6}$ These may indicate involvement of the AT2R in the physiology of adults and in the development and differentiation of fetal cells in the embryonic development stage.

\section{The AT2R Signaling Pathways}

The effects of AgII are mediated by a family of seven transmembrane receptors. In adult, the majority of the receptors are of the AT1R isoform, which is coupled to heterotrimeric $\mathrm{G}$ proteins $(\mathrm{Gq})$. AT2 receptor couples to

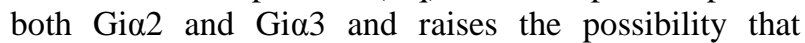
selective interactions between AT2R and different G proteins may result in specific cellular effects mediated by AT2 stimulation ${ }^{7}$. AT2R is a non classical GPCR for which $G$ protein coupling has not been demonstrated, but its conserved motif in the third intracellular loop may mediate novel intracellular effects. ${ }^{8}$

AT1R and AT2R-induced signals antagonize phosphorlipase $C-\beta$ phosphorylation. Ang II induced AT1R signals may be mainly blocked by AT2R signals through their negative cross-talk in the cytoplasm (at inositol phosphate production) rather than by the heterodimerization of both receptors on the cell surface. Thus, the proper balance of the expression levels of AT1R and AT2R might be critical for the antagonistic action between this receptors. ${ }^{9}$ 


\section{Roles of AT2R in cardiovascular functions}

While AT1R antagonists (ARBs) are well recognized and commonly used in the treatment of many cardiovascular disorders, the potential cardiovascular AT2R mediated effects are not well known.

\section{Role of AT2R in the control of vascular tone and arterial pressure}

The renin-angiotensin system is a major physiological regulator of body fluid volume, electrolyte balance, and arterial pressure. Virtually all of these actions of AgII have been attributed to an action at the AT1R. ${ }^{10}$ Different studies have revealed that AT2R mediate vascular relaxation, opposing the actions of AgII on AT1R.

An in vitro and in vivo study done on aortic and mesenteric vessels showed that AT2R stimulation by a non- peptide $\mathrm{C} 21$ causes a dose dependent vasorelaxation and evoked reduction in blood pressure. This vasorelaxation effect was abolished by AT2R antagonist, PD123319. The reduction of blood pressure was enhanced when combination of the AT1 receptor antagonist, candesartan, and $\mathrm{C} 21$ were given to hypertensive rats. ${ }^{11}$ The vasodilation of AT2R mediated effect has been also revealed by AT1R antagonist in the presence of an unopposed endogenous AgII. This implies that AT2R activation can facilitate the decrease in mean arterial pressure caused by an AT1 receptor antagonist, which was confirmed by the administration of cadesartan and AT2R agonist, CGP42112 to spontaneously hypertensive rats. ${ }^{12}$

The contribution of AT2R stimulation in vasodilation and antihypertensive effect produced by AT1R antagonists has also been demonstrated in rat isolated mesenteric resistance arterie. ${ }^{13}$ The increase in endogenous levels of Ang II during AT1R blockade may also contribute for the vasorelaxation effect in the presence of unblocked AT2R. Similarly, a study done in isolated uterine artery of pregnant mice demonstrated that AT2 receptor-mediated vasodilatation plays a role in modulating Ang-II contractile responses in pregnancy. ${ }^{14}$ The possible mechanisms of AT2R mediated vasodilation have been studied in isolated uterine artery of Sprague-Dawley rats that revealed AT2 receptor-mediated vasorelaxation via NO-cGMP pathway and a bradykinin $(\mathrm{BK}) \mathrm{B}_{2}$ receptor pathway (Figure 2). This mechanism had been confirmed by the reduction in contractile response of agiotensin II on uterine artery in the presence of HOE 140 (BK B2 antagonist), and No-nitro-L-arginine (NOLA) (NOS inhibitor). ${ }^{15}$ Another study demonstrated that stimulation of AT2 receptors caused a significant vasodilation through local production of BK in resistant arteries of rat mesentery in a flow-dependent manner. ${ }^{16}$ This effect had been shown by comparing the vasodilation response in kininogen negative rat with that of the wild one.

Moreover, another study demonstrated that AgII mediates renal production of $\mathrm{BK}$, which, in turn, releases nitric oxide and cGMP via stimulation of AT2 receptors. The authors also suggested that the increase in blood pressure and the decrease in renal $\mathrm{BK}$, nitric oxide, and cGMP during AT2R blockade indicate the roles of the AT2R in mediating the counter regulatory vasodilation effect in hypertension and prevention of a further increase in blood pressure. ${ }^{10}$ In addition, chronic infusion of $\mathrm{C} 21$ decreased nocturnal norepinephrine (NE) excretion and blood pressure via a nNOS/NO signaling pathway within paraventricular nucleus. ${ }^{17}$

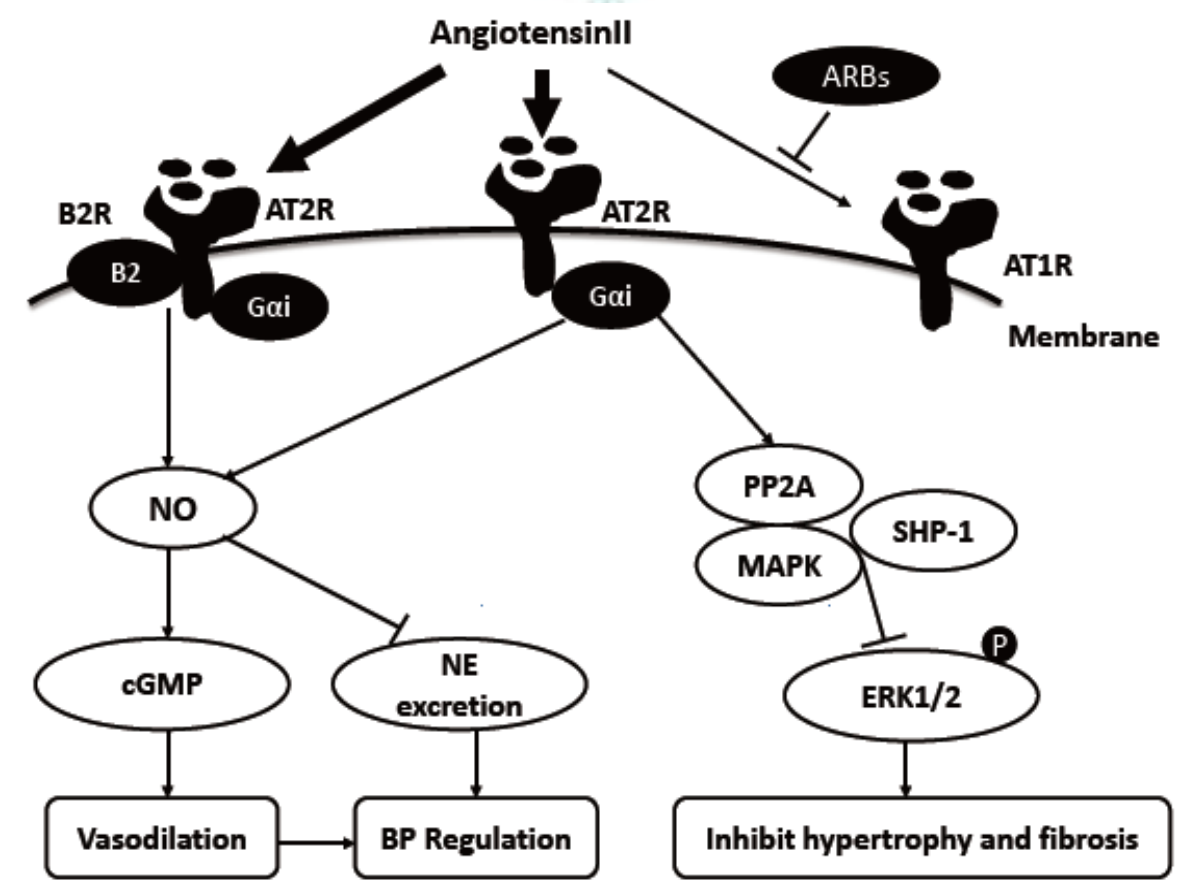

Figure 2: Putative mechanism underlying AT2R's function in cardiovascular diseases. ${ }^{18}$ Abbreviations:- AT2R, AgII Type-2 Receptor; AT1R, AgII Type-1 Receptor; ARBs, Angiotensin Receptor Blockers; B2R, Bradykinin B2 Receptor; Gai, G protein; NO, Nitric Oxide; cGMP, 3'-5'-cyclic guanosine monophosphate; NE, Norepinephrine; PP2A, Protein phosphatase 2A; MAPK, Mitogenactivated Protein Kinase; SHP-1, Src homology region 2 domain containing phosphatase; ERK1/2, Extracellular signal-regulated kinases 1 and 2 


\section{Effect of AT2Rs in the control of cardiovascular structure}

Both circulating and locally generated Ag II has contributed to remodeling after myocardial infarction developed by coronary ligation in pigs. Cardiac remodeling is reduced by the angiotensin converting enzyme inhibitors and AT1R blockers. It was suggested that the antihypertrophic effects of these drugs result not only from diminished AT1R stimulation but also from increased stimulation of growth-inhibitory AT2Rs. ${ }^{19}$

In addition, the antihypertrophic AT2R mediated effect was also shown in gene-targeted mice, which revealed an antihypertrophic effect in cardiac remodeling after myocardial cryo-injury and link the expression of cardiac endothelial nitric oxide synthase (eNOS) to AT2R activation. This has been indicated by an increased heart weight/body weight ratio, a significant low level of eNOS; accompanied by a decrease in cardiac cGMP levels in AT2R-deficient mice compared with control mice. ${ }^{20}$ Moreover, a AT2R gene transfer study in spontaneously hypertensive rats (SHR) revealed that AT2R over expression attenuates cardiac hypertrophy, independent of a change in blood pressure. $^{21}$ This beneficial outcome was marked by the decreased left ventricular wall thickness and heart weight to body weight ratio in AT2R treated SHRs compared with control animals.

The functional role of AT2R was also revealed in vivo in rat's heart in which AT2R played a role in the cardiac hypertrophic process by selectively regulating the expression of growth-promoting and growth-inhibiting factors. $^{22}$ This result had been shown by a marked increase in the gene expression of c-fos, endothelin-1, and insulin-like growth factor-1 and atrial natriuretic peptide in Ang II-induced hypertension when AT2R is blocked by PD123319. Contrary to the above studies, gene targeting in vivo study in mice indicated that left ventricular hypertrophy, impaired diastolic relaxation and cardiac fibrosis was higher in wild mice for AT2R than that of AT2R gene negative mice after Ang IIinfusion to induce hypertension. ${ }^{23,24}$

\section{Cardiac protective roles of AT2R in myocardial infarction}

Different studies demonstrated the AT2R mediated protective role after the development of myocardial infarction. For instance the cardio protective role of AT1R blocker, candesartan, was shown to be mediated by AT2R, which was conducted in pigs. ${ }^{25}$ Candesartan reduced the infarct size in treated pigs compared to the control groups. In addition, pretreatment with AT2R antagonist abolished the reduction of infarct size by candesartan, which indicates the involvement of AT2R in cardiac protection. Furthermore, C21 reduced the post infarction size in treated Wistar rats compared to the control via AT2R mediated anti--inflammatory and antiapoptotic effects. ${ }^{26}$ Another study conducted in AT2R transgenic mice also improves the left ventricular function after myocardial infarction compared to the wild type mice. The result showed a higher in the ejection fraction and a smaller end-systolic volume index (ESVI) in transgenic mice than that of wild type; but there was no difference in the size of the infarct. ${ }^{27}$ Related studies had shown that AT2 receptor deficiency exacerbates short-term death rates and heart failure after experimental acute myocardial infarction AT2R knockout mice. ${ }^{28,29}$ These findings indicate the protective roles of AT2R in cardiovascular functions with different mechanisms as illustrated in figure 3 .

\section{Effects of AT2R on renal system}

The renal AT1R and AT2R distribution was studied in the rat kidney using immune-histochemistry and RTPCR. AT1R translational sites were observed throughout the kidney, with the strongest labeling found in the vasculature of the renal cortex and the proximal tubules of the outer medulla. The AT2R protein expression was found throughout the rat kidney, although there was little to no expression found in the glomerulus and medullary thick ascending limbs of Henle (TAL). ${ }^{30}$ Another study revealed that AT2R was found on glomerular endothelial cells and tubular epithelial cells in the cortex, interstitial, and tubular cells in the outer medulla, and inner medullary collecting duct cells with no clear functions. ${ }^{31}$

\section{Roles in nephrogenesis}

As studied in the fetal lamb, both AT1R and AT2R were expressed early during nephrogenesis with specific spatial and temporal distribution during gestation. High-AT2R mRNA expression took place in the outermost nephrogenic area, in the undifferentiated mesenchymal cells and in differentiated epithelial cells of macula densa of maturing glomeruli. This may indicate that, AT2R in mesenchymal cells is involved in regulation of cell proliferation and apoptosis during the time of nephrogenesis. ${ }^{32}$ Another finding suggested that AT1R and AT2R are both involved in the modulation of development of the nephron, and that Ang II provides signals through both receptors. AT1R mainly stimulate cell proliferation but AT2R counter-regulates the AT1R mediated cell proliferation via inhibition of proliferation and apoptosis. ${ }^{33}$ The growth stimulating effect of AgII on embryonic renomedullary interstitial cells which is mediated by AT1R is also regulated by its antiproliferative effects mediated by AT2R. ${ }^{34}$

\section{AT2R mediated natriuresis effect}

The RAS plays various roles in the regulation of the homeostasis of blood composition and volume which in turn determines the blood pressure via AT1R. The renal role of AT2R in hypertension is not yet clear though different studies showed its natriuretic effect. A study conducted on the AT2R knockout mice showed that the blood pressure was higher in AT2 receptor knockout mice than control. At same renal perfusion pressure, wild-type mice excreted more sodium and water than AT2R knocked out mice. In addition, renal microsomes from AT2R knocked out mice had less activity in hydroxylating arachidonic acid than controls, which may contribute to the intrarenal differences. ${ }^{35}$ This can indicate the importance of AT2R in regulation of blood pressure by modulating intra renal diuresis effect. Similar study coducted in AT2R gene lacking mice after 
infusion of AgII was shown a marked and sustained increase in systolic blood pressure (SBP) and reduction in urinary sodium excretion, but neither SBP nor urinary sodium was changed in wild mice. In addition, the molecular mechanism of natriures effect of AT2R was related with the decrease in basal levels of renal interstitial fluid BK, and CGMP, an index of nitric oxide production in AT2R-null mice compared with wild mice. $^{36}$

The AT2R mediated natriuretic effect was also studied in the streptozotocin induced rats in which the tubular expression of AT2R and its natriuretic effect was higher in diabetic rats compared to the control rats. The natriuretic effect was reduced by the administration of AT2R antagonist, PD-123319, associated with a decrease in the level of cGMP; without any change in the glomerular filtration. ${ }^{37}$

The potential natriuresis effect of AT2R has also been observed by administering AT2R receptor agonist, C21, to obese Zucker rats. Infusion of $\mathrm{C} 21$ into rats under anesthesia caused a dose-dependent increase in urine flow and urinary Na volume which were blocked by preinfusion of the AT2R antagonist, PD123319. The excretion of sodium was not associated with the change in the glomerular filtration, indicating the tubular effect of C21. The diuresis and natriuresis effect was enhanced by the co-administration of amiliride, suggesting involvement of the AT2R. The involvement of AT2R in natriureis was associated with an increase in $\mathrm{NO}$ and cGMP. $^{38}$ All of the above effects of AT2R agonist revealed that AT2R is mediating natriuresis via NOcGMP pathway.

Moreover, the natriuresis effect of AT2R was potentiated by inhibition of $\mathrm{NAD}(\mathrm{P}) \mathrm{H}$ oxidase in a study conducted in Sprague-Dawley rats. Preinfusion of apocynin (NADPH oxidase inhibitor) had no effect on the net increase in urinary flow or urinary sodium rate in response to candesartan, however, it caused profound increases in CGP-42112A (AT2R agonist)-induced increase in urinary flow or urinary sodium rate and fractional excretion of Na. CGP-42112A infusion in the presence of apocynin increased urinary nitrite/nitrates and cGMP over basal, suggesting its signal transduction pathway. Based on the finding, NADPH oxidase may have an antagonistic role in AT2R mediated natriuresis/ diuresis possibly via neutralizing nitric oxide and thereby influence fluid-Na homeostasis. ${ }^{39}$

\section{Renal Protection Effects of AT2R}

The roles of AT2R in kidney disease protection are mediated via multitude of mechanisms, mainly by regulating the fibrosis, and inflammatory response as shown in the figure 3 . The anti-fibrotic and antiinflammatory effects mediated by AT2R were observed in a study conducted in AT2R gene null mice with kidney disease induced by unilateral ureteral obstructtion. ${ }^{40}$ The result of this study revealed that more severe fibrosis (with increased collagen) in interstitial cells of kidney of AT2R null mice was observed compared to the wild mice. In addition, though cell proliferation and macrophage infiltration were comparable, an increased level of fibroblasts and myofibroblasts were present in obstructed kidney of the AT2R null mice compared to that of wild mice. This indicates the protective role of AT2R in the remodeling of the kidney cell following an injury. The result of another study is consistent with the above study in which the role of AT2R was studied by administering PD-123319. Treatment with PD-123319 was found to exacerbate the increase in interstitial volume and collagen IV matrix score of the ureteral obstructed kidney. ${ }^{41}$ These results suggest that AgII operating through the AT2R exerts an antifibrotic effect on the kidney during obstructive nephropathy in opposition to the profibrotic effects of AgII acting through the AT1R. Interestingly, the AT2R agonist, CGP-42112A treatment in obese rats reduced the plasma and kidney cortex inflammatory (TNF- $\alpha$, IL-6) and oxidative stress (gp-91 ${ }^{\text {phox }}$ ) markers and increased plasma antioxidant activity to the levels seen in lean control rats. ${ }^{39}$ This indicates the importance of AT2R in reducing renal inflammation related to obesity caused hypertension.

As mentioned earlier, the anti-inflammatory effects of renal proximal tubule AT2R has been associated with the increase in the anti-inflammatory interleukin-10 as studied in vivo and in vitro studies. ${ }^{42}$ Protective role of the AT2R in a renal wrap hypertension has been shown in one kidney wrap model of rats. In this study, PD 123319, a specific AT2-receptor antagonist, significantly increased SBP and decreased renal interstitial fluid (RIF), BK, nitric oxide metabolites (NOX), and cGMP in both kidneys. In contrast, losartan normalized SBP and increased renal function, RIF BK, NOX, and cGMP in contralateral kidneys. ${ }^{36}$

\section{Role of AT2R on Nervous System}

The AT2R is not only limited to peripheral tissues, but also found in the brain with wide distribution. AT1R receptor subtypes in the brain is present in areas related to regulation of blood pressure, water intake (circumventricular organs and paraventricular nucleus), and pituitary hormone secretion, and AT2R is found in different brain areas of which function has not been yet determined.

According to an in vitro study done from brain of adult and two week old rats, AT2R has been detected in lateral septum, subthalamlic nucleus, and inferior olive in both rats. AT2R was also found in thalamic nuclei, the nuclei of the $3^{\text {rd }}$ and $12^{\text {th }}$ cranial nerves, geniculate bodies, cerebellum, and cingulated cortex in brain of young rats. ${ }^{44}$ In addition, AT2R have been found in somato-dendrite parts of hypothalamus-paraventricular neurons expressing vasopressin and other neuromodulators with a probable strategic position for surface activation by Ag II and/or intracellular trafficking. ${ }^{45}$ The increased expression of AT2R has been shown in hypothalamus in the SHR compared to that of the normotensive Wistar- Kyoto rat (WKY) ${ }^{46}$, suggesting the hyperactive RAS system in pathologic conditions. AT2R is also localized in sensory neurons of human dorsal root ganglion, and nerve fibers in peripheral nerves, skin, urinary bladder and bowel ${ }^{47}$ which may indicates its involvement in nociception. 
On top of these, a comparative in vitro study revealed that astrocyte cultures from neonatal rat brains contain predominantly AT1R that are coupled to a stimulation of inosito-phospholipid hydrolysis. In contrast, neuron cultures contain mostly AT2R that are coupled to a reduction in basal cGMP levels, but a smaller population of AT1R is also present in these neurons. ${ }^{48}$

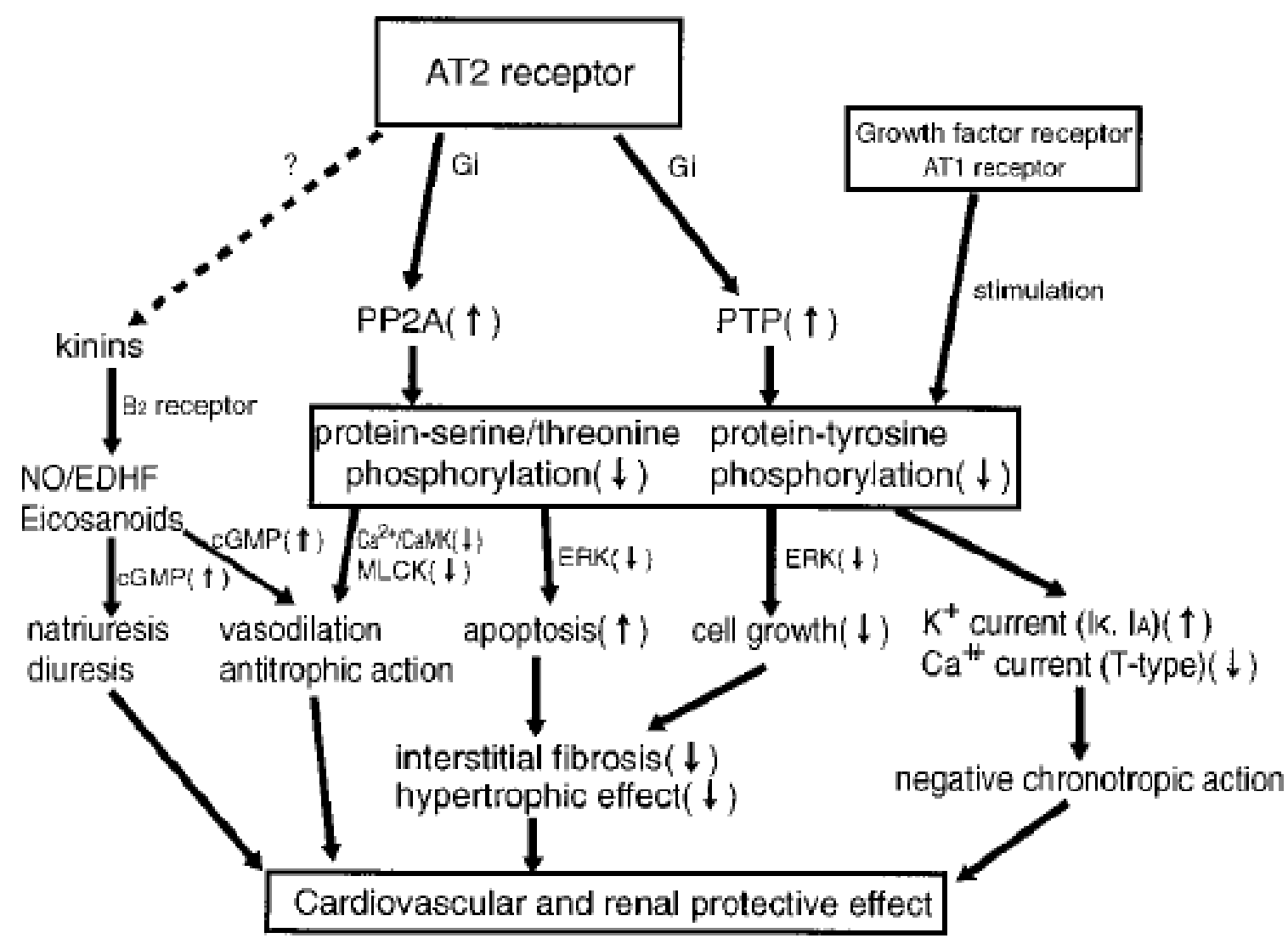

Figure 3: Proposed AT2-R-mediated effects on cardiovascular and renal system. ${ }^{43} \mathrm{PP} 2 \mathrm{~A}$ indicates serine/threonine phosphatase $2 \mathrm{~A}$; $I \mathrm{k}$, delayed rectifier $\mathrm{K} 1$ current; IA, transient outward $\mathrm{K} 1$ current; $\mathrm{Ca}^{+2} / \mathrm{CaMK}$; calcium calmodulin kinase; MLCK, myosin light chain kinase; and EDHF, endothelium-derived hyperpolarizing factor.

\section{Neurite outgrowth and differentiation}

Functional studies in neuronal cells have demonstrated the capability of AT2R to modulate neuronal excitability, neurite elongation, and neuronal migration, which may be an important regulator of brain functions. ${ }^{49}$ An in vitro study conducted on cerebellar cell cultures revealed that AT2R activation by AgII enhanced cell differentiation, and involved in neurite outgrowth and cell migration (morphological differentiations), which are important processes in the organization of the various layers of the cerebellum. Activation of AT2R by Ag II, in the presence of AT1R antagonist, has been associated with increased elongation of neurites and cell migration from the edge of the micro-explant toward the periphery. In addition, there were an increase in the expression of neuronspecific bIII-tubulin, and expression of the microtubuleassociated proteins tau and MAP2 (proteins stabilizing the polymerized microtubules), indicating the involvement of AT2R in neuronal cell differentiation. These effects have been abolished when Ang II was coincubated with PD-123319 (AT2R antagonist). ${ }^{50}$ In addition, this study is consistent with another study showing the increase in neurite length and density when
AT2R on dorsal ganaglionic neurons are stimulated by AgII. ${ }^{51}$

\section{Neuroprotection}

The functional role of AT2R in the prevention of neuronal injury related to stroke and ischemia has been elucidated by different investigations. A study conducted in AT2R negative and wild type mice demonstrated that AT2R stimulation has a protective effect on ischemic brain lesions, at least partly through the modulation of cerebral blood flow and superoxide production. After ischemia is induced by middle cerebral artery occlusion, the ischemic area, the neurological deficit was significantly larger in AT2R-deficient mice than in wild type mice. Superoxide production and NADPH oxidase activity were enhanced in the ischemic area of the brain in the receptor deficient mice. Moreover, an AT1R blocker, valsartan, significantly inhibited the ischemic area, neurological deficit, and reduction of cerebral blood flow as well as superoxide production and NADPH oxidase activity in wild type mice, but these effects of valsartan were weaker in AT2R deficient ones. ${ }^{52}$ Moreover, AT1 Receptor blockade promotes the local AgII System in cerebral micro vessels in SHR compared to the control rats. The increased AT2R 
expression and stimulation after administration of candesartan may contribute to the protection against brain ischemia and inflammation. ${ }^{50,51}$

The direct neuroprotective effect of AT2R stimulation by intra cerebroventricularly administration of CGP42112 before stroke induction has been revealed by another study conducted in spontaneously hypertensive mice (SHM). Based on infarct, and behavioral data, CGP42112 dose-dependently reduced cortical infarct volume and motor deficit. In addition, there was an increased neuronal survival in the infarcted region. ${ }^{53}$ similarly, centrally administered C21 to SMH before and after induction of stroke by middle cerebral endothelin confers neuroprotection against stroke damage. ${ }^{54}$ This benefit has been suggested to be likely to involve various mechanisms, including microglial activation of endogenous repair and enhanced cerebroperfusion. Similarly, in vitro study showed that AgII protected neuronal ischemic injury by activating AT2R in the cultured cortical neurons. After oxygen-glucose ischemia/reperfusion model, Ang II ameliorated the neuronal injury caused by oxygen-glucose deprivation and also increased cell viability through inhibiting cell apoptosis by reversing the increased Bax mRNA and the decreased $B c l 2$ mRNA expression. These protective activities of Ang II has been attenuated by co-treatment with AT2R blocker (PD123319) ${ }^{55}$, indicating the role of AT2R in neuroprotection after injury.

The molecular mechanism of AT2R mediated neuroprotection may be related to the increased level of a neuroprotective factor, methyl methanesulfonate sensitive 2 (MMS2) as described by study in mice. According to this study, after mice underwent permanent middle cerebral artery occlusion, AT2R mRNA expression was significantly increased in the ischemic side of the brain. In ischemic brain regions, MMS2 was increased in wild-type mice, but not in AT2R knockout mice. This has been confirmed by administering Valsartan which enhanced MMS2 expression to a greater extent in wild-type mice compared to the AT2R knockout mice. To confirm the role of MMS2, intracerebroventricular administration of MMS2 siRNA showed more impaired avoidance rate after middle cerebral artery occlusion compared with that in control siRNA-transfected mice. ${ }^{56}$ This direct relationship between AT2R and MMS2 expression gives a clue for signal transduction of AT2R stimulation for neuroprotection via DNA repair mechanism following ischemia. In addition, the proangiogenic effect of AT1R antagonist in the endothelial cells of micro vessels in the brain may be related to the expression of BDNF via indirect AT2R stimulation by Ang II. ${ }^{57}$

\section{Cognitive function}

The neurodegenerative effect of AT2R oligomers has been described by a study using animal models of Alzheimer disease (AD). Neurodegeneration in AD correlates with dysfunction of diverse signal transduction mechanisms, such as the G-protein-stimulated phosphoinositide hydrolysis mediated by Gaq. It was stated that impaired Gaq-stimulated signaling in brains of $\mathrm{AD}$ patients and mice correlated with the appearance of cross linked oligomeric AT2R, sequestering Gaq. Amyloid $\beta(\mathrm{A} \beta)$ was causal to AT2R oligomerization, because cerebral microinjection of A $\beta$ triggered AT2R oligomerization in the hippocampus of mice in a dosedependent manner. A $\beta$ induced AT2 oligomerization by a two-step process of oxidative and transglutaminasedependent cross-linking. The induction of AT2 oligomers in a transgenic mouse model with AD-like symptoms was associated with Gaq dysfunction and enhanced neurodegeneration. Vice versa, stereotactic inhibition of AT2 oligomers by RNA interference prevented the impairment of Gaq and delayed Tau phosphorylation. Thus, $\mathrm{A} \beta$ induces the formation of cross-linked AT2 oligomers that contribute to the dysfunction of Gaq in an animal model of Alzheimer disease. ${ }^{58}$ This can lead to dysfunction of the cognitive function of the brain.

In addition, the cognitive function of AT2R has been shown by neuroprotective study in the case of ischemic neuronal injury by using passive avoidance test to relate neuroprotection with cognitive function. According to this study, cognitive function was significantly impaired in AT2R null mice compared to that of the wild type mice. Treatment with valsartan prevented the cognitive decline in wild-type mice, but this effect was weaker in AT2R null mice. ${ }^{56}$

\section{CONCLUSION}

AT2R mediates different regulatory effects in the cardiovascular, renal and nervous system through its anti-inflammatory, anti- proliferative, vasodilator and natriuresis actions, most of which are counter regulatory to the actions mediated by AT1R. The vasodilation, natriuresis and anti-inflammatory effects are very important actions to regulate and control the rise in blood pressure and the subsequent complications on the heart, blood vessels, kidney, brain and other end organs. Thus, the AT2R mediates the protective effects in cardiac, renal and brain injury induced by different factors. Most of the aforementioned actions mediated by AT2R involve the BK and/or NO-cGMP signal transduction pathways and accompanied by the production of inflammation protective factors like interleukin-10 and neuroprotective factors like MMS2 and BDNF. Therefore, the development of AT2R agonist can be a potential target in the principle of prevention and treatment of hypertension, myocardial infarction, stroke and other organ diseases. In addition, further studies have to be conducted that can show the molecular signal transduction involving effects mediated by the AT2R.

Funding: This review received no specific grant from any funding agency

Competing interests: The authors declare that there is no competing interest 


\section{REFERENCES}

1. Padia SH., Carey RM., AT2 receptors: Beneficial counterregulatory role in cardiovascular and renal function. European Journal of Physiolog 2013; 465: 99-110.

2. Wright JW., Kawas LH., Harding JW., A role for the brain RAS in Alzheimer's and Parkinson's diseases. Frontiers in endocrinology 2013; 3:158.

3. De Gasparo M., Catt KJ. Inagami T., Wright JW, Unger T., International Union of Pharmacology. XXIII. The Angiotensin II Receptors. Pharmacology Review 2000; 52:415-472.

4. Steckelings UM., Rompe F., Kaschina E., Namsolleck P., Grzesiak A., Funke-Kaiser H., et al.,The past, present and future of angiotensin II type 2 receptor stimulation. Journal of Renin-Angiotensin-Aldosterone System 2010; 11:67-73.

5. Tsutsumi Y, Matsubara H, Ohkubo N, Mori Y, Nozawa Y, Murasawa S, et al., Angiotensin II Type 2 Receptor Is Upregulated in Human Heart With Interstitial Fibrosis, and Cardiac Fibroblasts Are the Major Cell Type for Its Expression. Circulation Research 1998; 83: 1035-1046.

6. Yu L., Zheng M., Wang W., Rozanski GJ., Zucker IH, Gao L., Developmental changes in AT1 and AT2 receptor-protein expression in rats. Journal of Renin Angiotensin Aldosterone System 2011; 11: 214-221.

7. Zhang J., Pratt RE., The AT2 Receptor Selectively Associates with Gia2 and Gia3 in the Rat Fetus. The Journal of Biological Chemistry 2010; 271: 15026-15033.

8. Mukoyama M., Nakajima M., Horiuchi M., Sasamura H., Pratt RE., Dzau VJ., Expression Cloning of Type 2 Angiotensin II Receptor Reveals a Unique Class of Seven-transmembrane Receptors. The Journal of Biological Chemistry 1993; 268 24539-24542.

9. Miuraa S., Matsuoa Y., Kiyaa Y., Karnikb SS., Sakua K.., Molecular mechanisms of the antagonistic action between AT1 and AT2 receptors. Biochemical and Biophysical Research Communications 2010; 391: 85-90.

10. Carey RM, Wang Z, Siragy HM. Role of the Angiotensin Type 2 Receptor in the Regulation of Blood Pressure and Renal Function. Hypertension 2000, 35:155-163

11. Bosnyak S, Welungoda IK, Hallberg A, Alterman M, Widdop RE, Jones ES. Stimulation of angiotensin AT2 receptors by the non-peptide agonist, Compound 21, evokes vasodepressor effects in conscious spontaneously hypertensive rats. British Journal of Pharmacology 2010, 159: 709-716

12. Barber MN, Sampey DB, Widdop RE. AT2 Receptor Stimulation Enhances Antihypertensive Effect of AT1 Receptor Antagonist in Hypertensive Rats. Hypertension 1999, 34: 1112-1116

13. Widdop RE, Matrougui K, Levy BI, Henrion D. AT2 Receptor-Mediated Relaxation is Preserved After Long-Term AT1 Receptor. Hypertension 2002, 40:516-520.

14. Pulgar VM, Yamashiro H, Rose JC, Moore LG. Role of the AT2 receptor in modulating the angiotensin-II contractile response of the uterine artery at mid gestation. Journal of Renin Angiotensin Aldosterone System 2011, 12: 176-183.

15. Hannan RE, Davis EA, Widdop RE. Functional role of angiotensin II AT2 receptor in modulation of AT1 receptormediated contraction in rat uterine artery: involvement of bradykinin and nitric oxide. British Journal of Pharmacology 2003, 140: 987-995.

16. Katada J, and Majima M. AT2 receptor-dependent vasodilation is mediated by activation of vascular kinin generation under flow conditions. British Journal of Pharmacolog 2002, 136: 484-491

17. Gao L, Zucker IH. AT2 receptor signaling and sympathetic regulation. Current Opinion Pharmacology 2011, 11:124-130.

18. Li Y, Li X, Yuan H. Angiotensin II type-2 receptor-specific effects on the cardiovascular system. Cardiovascular Diagnosis and Therapy 2012, 2: 56-62.

19. Kats JP, Duncker DJ, Haitsma DB, Schuijt MP, Niebuur R, Stubenitsky $\mathrm{R}$, et al. Angiotensin- converting Enzyme Inhibition and Angiotensin II Type 1 Receptor Blockade Prevent Cardiac Remodeling 2000

20. Brede M, Roell W, Ritter O, Wiesmann F, Jahns R, Haase A, et al. Cardiac Hypertrophy Is Associated with Decreased
eNOS Expression in Angiotensin AT2 Receptor-Deficient Mice. Hypertension 2003, 42: 1177-1182.

21. Metcalfe BL, Huentelman MJ, Parilak LD, Taylor DG, Katovich MJ, Knot HJ, et al . Prevention of Cardiac Hypertrophy by Angiotensin II Type-2 Receptor Gene Transfer. Hypertension 2004, 43:1233-1238

22. Lakó-Futó Z, Szokodi I, Sármán B, Földes G, Tokola H, Ilves M, et al. Evidence for a Functional Role of Angiotensin II Type 2 Receptor in the Cardiac hypertrophic Process in Vivo in the Rat Heart. Circulation 2003, 108: 2414-2422.

23. Ichihara S, Senbonmatsu T, Jr EP, Ichiki T, Gaffney FA, Inagami T. Angiotensin II Type 2 Receptor: Is Essential for Left Ventricular Hypertrophy and Cardiac Fibrosis in Chronic Angiotensin II -Induced Hypertension. Circulation 2001, 104: 346-35

24. Jehle AB, Xu Y, DiMaria JM, French BA, Epstein FH, Berr SS, et al. A Non-peptide Angiotensin II Type 2 Receptor Agonist Does Not Attenuate Post-Myocardial Infarction Left Ventricular Remodeling in Mice. Journal of Cardiovascular Pharmacology 2012, 59: 363-368.

25. Jalowy A, Schulz R, Dörge H, Behrends M, Heusch G. Infarct Size Reduction by AT1-Receptor Blockade Through a Signal Cascade of AT2-Receptor Activation, Bradykinin and Prostaglandins in Pigs. Journal of American College of Cardiology 1998, 32:1787-96.

26. Kaschina E, Grzesiak A, Li J, Foryst-Ludwig A, Timm M, Rompe F, et al. Angiotensin II Type 2 Receptor Stimulation: A Novel Option of Therapeutic Interference With the ReninAngiotensin System in Myocardial Infarction? Circulation 2008, 118: 2523-2532.

27. Yang Z, Bove CM, French BA, Epstein FH, Berr SS, DiMaria $\mathrm{JM}$, et al . Angiotensin II Type 2 Receptor Overexpression Preserves Left Ventricular Function After Myocardial Infarction. Circulation 2002, 106:106-111.

28. Adachi,Y, Saito Y, Kishimoto I, Harada M, Kuwahara K, Takahashi N, et al. Angiotensin II Type 2 Receptor Deficiency Exacerbates Heart Failure and Reduces Survival after Acute Myocardial Infarction in Mice. Circulation 2003, 107: 24062408

29. Oishi Y, Ozono R, Yano Y, Teranishi Y, Akishita M, Horiuchi M, et al. Cardioprotective Role of AT2 Receptor in Postinfarction Left Ventricular Remodeling. Hypertension 2003, 41: 814-818.

30. Miyata N, Park F, Li XF, Cowley AW. Distribution of angiotensin AT1 and AT2 receptor subtypes in the rat kidney. American Journal of Physiology 1999, 277: F437-F446.

31. Wehbi GJ, Zimpelmann J, Carey RM., Levine DZ., Burns KD. Early streptozotocin-diabetes mellitus downregulates rat kidney AT2 receptors. American Journal of Physiology 2001, 280: F254-F265

32. Gimonet V, Bussieres L, Medjebeur AA, Gasser B, Lelongt B, Laborde K.. Nephrogenesis and angiotensin II receptor subtypes gene expression in the fetal lamb. American Journal of Physiology 1998, 274: F1062-F1069.

33. Wolf G. Angiotensin II and tubular development. Nephrology Dialysis Transplantation 2002, 17: 48-51.

34. Maric C, Aldred GP, Harrris PJ, Alcorn D. Angiotensin II inhibits the growth of cultured embryonic renomedullary interstitial cells through AT2R. Kidney international 1998, 53:92-99.

35. Gross V, Schunck W, Honeck H, Milia AF, Rgel EK, Walther $\mathrm{T}$, et al. Inhibition of pressure natriuresis in mice lacking the AT2 receptor. Kidney International 2000, 57:191-202.

36. Siragy HM, Inagami T, Ichiki T, Carey RM. Sustained hypersensitivity to angiotensin II and its mechanism in mice lacking the subtype-2 (AT2) angiotensin receptor. Proceeding national academy for science 1999, 96: 6506-6510.

37. Hakam AC, Siddiqui AH, Hussain T. Renal angiotensin II AT2 receptors promote natriuresis in streptozotocin-induced diabetic rats. American Journal of Physiology- Renal Physiology 2006, 290: F503-F508. 
38. Ali Q and Hussain T. AT2 receptor non-peptide agonist C21 promotes natriuresis in obese Zucker rats. Hypertension Research 2012, 35: 654-660.

39. Sabuhi R, Ali Q, Asghar M, Al-Zamily NRH, Hussain T. Role of the angiotensin II $\mathrm{AT}_{2}$ receptor in inflammation and oxidative stress: opposing effects in lean and obese Zucker rats. American Journal of Physiology- Renal Physiology 2011, 300: F700-F706.

40. Ma J, Nishimura H, Fogo A, Kon V, Inagami T, Ichikawa I. Accelerated fibrosis and collagen deposition develop in the renal interstitium of angiotensin type 2 receptor null mutant mice during ureteral obstruction. Kidney international 1998, 53: 937-944.

41. Morrissey JJ, Klah S. Effect of AT2 receptor blockade on the pathogenesis of renal fibrosis. American journal of physiology 1999, 276: F39-F45.

42. Dhande I, Ali Q, Hussain T. Proximal tubule angiotensin at2 receptors mediate an anti-inflammatory response via interleukin-10: role in renoprotection in obese rats. Hypertension 2013, 61: 1218-1226.

43. Matsubara H. Pathophysiological Role of Angiotensin II Type 2 Receptor in Cardiovascular and Renal Diseases. Circulation research 1998, 83: 1182-1191.

44. Millan MA, Jacobowitz DM, Aguilera G, Catt KJ. Differential distribution of AT1 and AT2 angiotensin II receptor subtypes in the rat brain during development. Proceeding National Academy for Science 1991, 88: 11440-11444.

45. Coleman CG, Anrather, J, Iadecola C, Pickel VM. Angiotensin II type 2 (AT2) receptors have a major somatodendritic distribution in vasopressin-containing neurons in the mouse hypothalamic paraventricular nucleus. Neuroscience 2009, 163: 129-142.

46. Han NL, Sim MK. Hypothalamic angiotensin receptor subtypes in normotensive and hypertensive rats. American Journal of Physiology 1998, 275: H703-H709.

47. Anand U, Facer P, Yiangou Y., Sinisi M, Fox M, McCarthy T, et al. Angiotensin II type 2 receptor (AT2R) localization and antagonist-mediated inhibition of capsaicin responses and neurite outgrowth in human and rat sensory neurons. European Journal of Pain 2013, 17: 1012-1026.

48. Sumners C, Tang W, Zelezna B, Raizada MK.. Angiotensin II receptor subtypes are coupled with distinct signal-transduction mechanisms in neurons and astrocytes from rat brain. Proceeding National Academy for Science 1991, 88:75677571.
49. Gendron L, Payet, MD, Gallo-Payet N. The angiotensin type 2 receptor of angiotensin II and neuronal differentiation: from observations to mechanisms. Journal of Molecular Endocrinology 1991, 31, 359-372.

50. Co^ te’ F, Do TH, Laflamme L, Galloi J, Gallo-Payet N. Activation of the AT2 Receptor of Angiotensin II Induces Neurite Outgrowth and Cell Migration in Microexplant Cultures of the Cerebellum. The journal of biological chemistry 1999, 274: 31686-31692.

51. Anand U, Facer P, Yiangou Y., Sinisi M, Fox M, McCarthy T, et al. Angiotensin II type 2 receptor (AT2R) localization and antagonist-mediated inhibition of capsaicin responses and neurite outgrowth in human and rat sensory neurons. European Journal of Pain 2013, 17: 1012-1026.

52. Iwai M, Liu H, Chen R, Ide A, Okamoto S, Hata R, et al. Possible Inhibition of Focal Cerebral Ischemia by Angiotensin II Type 2 Receptor Stimulation. Circulation 2004, 110: 843848.

53. McCarthy CA, Vinh A, Callaway JK, Widdop RE. Angiotensin AT2 Receptor Stimulation Causes Neuroprotection in a Conscious Rat Model of Stroke. Stroke 2009, 40: 1482-1489

54. McCarthy CA, Vinh A, Miller AA, Hallberg A, Alterman M, Callaway JK, et al. Direct Angiotensin AT2 Receptor Stimulation Using a Novel AT2 Receptor Agonist, Compound 21, Evokes neuroprotection in Conscious Hypertensive Rats. Public library of science 2014, 9: e95762.

55. Tang M, Zhao L, Chen Y, Wang L, Zhang X. Angiotensin II protects cortical neurons against oxygen-glucose deprivation-induced injury in vitro. Biomedical reports 2014, 2: $112-116$.

56. Mogi M, Li J, Iwanami J, Min L, Tsukuda K, Iwai M, et al. Angiotensin II Type-2 Receptor Stimulation Prevents Neural Damage by Transcriptional Activation of Methyl Methanesulfonate Sensitive 2. Hypertension 2006, 48: 141-148

57. Alhusban A, Kozak A, Ergul A, Fagan SC. AT1 Receptor Antagonism Is Proangiogenic in the Brain: BDNF a Novel Mediator. Journal of Pharmacology and Experimental Therapeutics, 2013

58. Abdalla S, Lother H, Missiry A, Langer A, Sergeev P, Faramawy Y, et al. Angiotensin II AT2 Receptor Oligomers Mediate G-protein Dysfunction in an Animal Model of Alzheimer Disease. Journal of Biological Chemistry 2009, 284: 6554-6565. 